\title{
Model-Based Part Manufacturing Quality Inspection Path Planning
}

\author{
Zhihua Zhang $\mathbb{D}^{1}{ }^{1}$ Amar Jain, ${ }^{2,3}$ and Vinay Kumar ${ }^{4}$ \\ ${ }^{1}$ School of Mechano-Electronic Engineering, Xidian University, Xi'an, 710071 Shaanxi, China \\ ${ }^{2}$ Research Scholar, Department of Civil Engineering, Faculty of Engineering and Technology, Madhyanchal Professional University, \\ Bhopal, India \\ ${ }^{3}$ Sanskriti University, Mathura, India \\ ${ }^{4}$ Department of Computer Engineering and Application, GLA University, Mathura, India
}

Correspondence should be addressed to Zhihua Zhang; jwzhang_2@stu.xidian.edu.cn

Received 13 December 2021; Accepted 2 February 2022; Published 24 February 2022

Academic Editor: Deepak Kumar Jain

Copyright (C) 2022 Zhihua Zhang et al. This is an open access article distributed under the Creative Commons Attribution License, which permits unrestricted use, distribution, and reproduction in any medium, provided the original work is properly cited.

\begin{abstract}
This article mainly studies the path planning of part manufacturing quality inspection based on models. Therefore, this paper optimizes the inspection path planning by combining the deep learning of the BP neural network in the neural network model, then improves the recognition efficiency of parts with various shapes through the collection of surface point information, and then combines the basic principles of model inspection and quality control principles to improve the accuracy of quality inspection. In order to better design this optimal path, this paper also designs welding basic formation parameter experiments and robustness analysis experiments to verify the influencing factors of the welding process and the specific results of image processing; this paper also designed the part outer diameter quality inspection test analysis to verify the accuracy and coverage of model-based part manufacturing quality inspection. The results obtained through the collection of experiments are finally compared with the traditional part quality inspection path; the experimental results show that compared with the traditional part quality inspection path, the new part quality inspection path can improve the accuracy rate of 5\%-17\%, the coverage rate of $9 \%-20 \%$, and efficiency of 3\%-17\%.
\end{abstract}

\section{Introduction}

Geometric measurement has been widely used in the production of parts, and the improvement of the level of geometric measurement is conducive to ensuring the quality of parts. With the increasing application of artificial neural network (ANN) models in the manufacturing field, geometric measurement based on ANN models has also received more and more attention. The study of geometric measurement based on the ANN model is conducive to the integration of design, measurement, and processing, thereby improving the efficiency of part processing, and lays a foundation for the development of measurement technology [1].

In recent years, artificial neural networks have become a research hotspot in many fields. With the development of artificial neural networks, visual inspection technology is widely used in many fields, especially products having the advantages of noncontact, high precision, and high speed, defect detection, and product size inspection field [2]. The quality inspection of parts is an important link that must be carried out after the parts are processed. The screw defects on the surface of the bolts and the dimensional inspection of the main parts of the valve are still used in visual form, and the inspection efficiency is improved through quality inspection or conventional inspection tools.

In some domestic manufacturing industries, manual testing, a pure operation method, occupies a large part, which is inefficient and does not effectively combine product quality and industrial computer network control. Customers measure and select products and suppliers, and quality has gradually become the most important benchmark. This article is aimed at part manufacturing, assembly, and test production line, because in the mass production process, there are often workers' omissions, misflows, etc., which 
affect the quality of the final product to be systematically improved. Build a local area network within the enterprise, establish a system database between the client and the server, upload relevant test information to the library system by binding barcodes, and realize the chain error prevention function for the monitoring of the assembly line test line process. Strictly guarantee the quality of the products. Regular and effective maintenance and management of the server, in the process of actual production and application, the reliability, and stability of the system are greatly guaranteed, so this article combines artificial neural networks to optimize the part quality inspection path of the model.

Zheng and $\mathrm{Wu}$ proposed an intelligent spare part inventory management system. They divide the spare parts into consumables and spare parts according to the characteristics of spare parts and manage them through the cyberphysical inventory management system [3]. It studies the inventory management system of semiconductor manufacturing, but this article mainly studies part manufacturing. Maria is researching $3 \mathrm{D}$ printing to additively manufacture printed spare parts [4]. He is researching $3 \mathrm{D}$ printing material manufacturing, but this article is researching quality monitoring of part manufacturing. In order to study the function of the hippocampus, Chen proposed a neural network model of hippocampus-cortex interaction. With the help of a comprehensive calculation model of cortical processing, he considered the correlation between hippocampal dysfunction and schizophrenia symptoms [5]. He uses the neural network model to study the function of the hippocampus, which has a certain reference value for this article, but it is not great. The ability to recognize and predict sensory input time series is critical to survival in the natural environment. Cui et al. analyzed the characteristics of HTM sequence memory and applied it to the sequence learning and prediction problems of streaming data [6]. They designed a model to recognize and predict sensory input time series, but what this article needs to design is an optimized path model for part monitoring. In order to study the solar radiation model, Truong et al. proposed an ANN model for estimating global solar radiation in the eastern Mediterranean region of Turkey. They compared the estimated results of the model with the measured data at four locations [7]. The ANN model they studied is applied to solar radiation, and this article is mainly applied to the optimization path of part manufacturing quality monitoring. Wang et al. established a data-driven model for battery state-of-charge estimation. They built a neural network model for battery energy state estimation. The battery state estimation adopts the estimation method based on probability [8]. Water quality testing is essential for guiding water safety management and ensuring public health. Peletz et al. research to strengthen the regulated water quality testing program to provide evidence [9]. They mainly based on the quality monitoring of water quality, and this article mainly studies the quality monitoring of parts. In order to explore the application of AIS in route planning, Naus described a method of drafting route plan templates based on historical data of AIS (Automatic Identification System) [10]. The path planning method he studied has a certain reference value for this article, but it has little effect.
Most of the documents cited in this article are about the quality inspection of parts, but they lack the application of path optimization. This is where this article needs to think about.

The innovation of this article is to combine the BP neural network model in the ANN to optimize the part quality inspection path and then combine the surface point collection information extraction, the basic principles of model inspection, and the quality control principle to improve accuracy, coverage, and efficiency. And through welding basic formation parameter experiment, robustness analysis experiment, and part outer diameter quality inspection test to collect data, the advantages of the new part quality inspection path are proved compared with the traditional part quality inspection path.

\section{Part Manufacturing Inspection Method Based on Neural Network Model}

2.1. ANN Model. With the continuous advantages of mechanical learning [11] in the technical field and the importance of partial monitoring in this article, it has gradually played an important role in the quality monitoring stage [12]. Figure 1 shows the application of ANNs.

2.1.1. RNN Recurrent Neural Network. Due to the time correlation and overall logic characteristics of time series data [13], traditional neural network information is one-way propagation. Therefore, unlike the training or use of a fully connected neural network or a folded neural network, in some specific data, the current neural network [14] (referred to as RNN) is based on the usual multilayer BP neural network to improve the level of the unit.

Like the traditional neural network, the current neural network (RNN [15]) includes an input layer, a hidden layer, and an output layer. The hidden layer is assigned several weight matrices and optimized using the optimization function [16, 17] (loss function). The current neural network (RNN) has introduced the following two innovations.

(1) Assign weights to both real-time input and historical input and confirm the final weight through the optimization function

(2) Imported antiattribute algorithm (BPTT for short). The current neural network (RNN) can inherit the traditional gradient descent algorithm to optimize the network model [18] and use the commonly used probability gradient descent algorithm to optimize the current neural network (RNN) model

2.1.2. BP Neural Network. ANN is a model for scientists to analyze and process information by simulating the human brain and nervous system based on the basic principles of biological neural networks.

The structure of an ANN model is a simulation of the structure of brain neurons. An ANN model is mainly composed of $n$ nodes and directed edge connections. Each node is a simulation of brain neurons, and a directed edge is a pair 


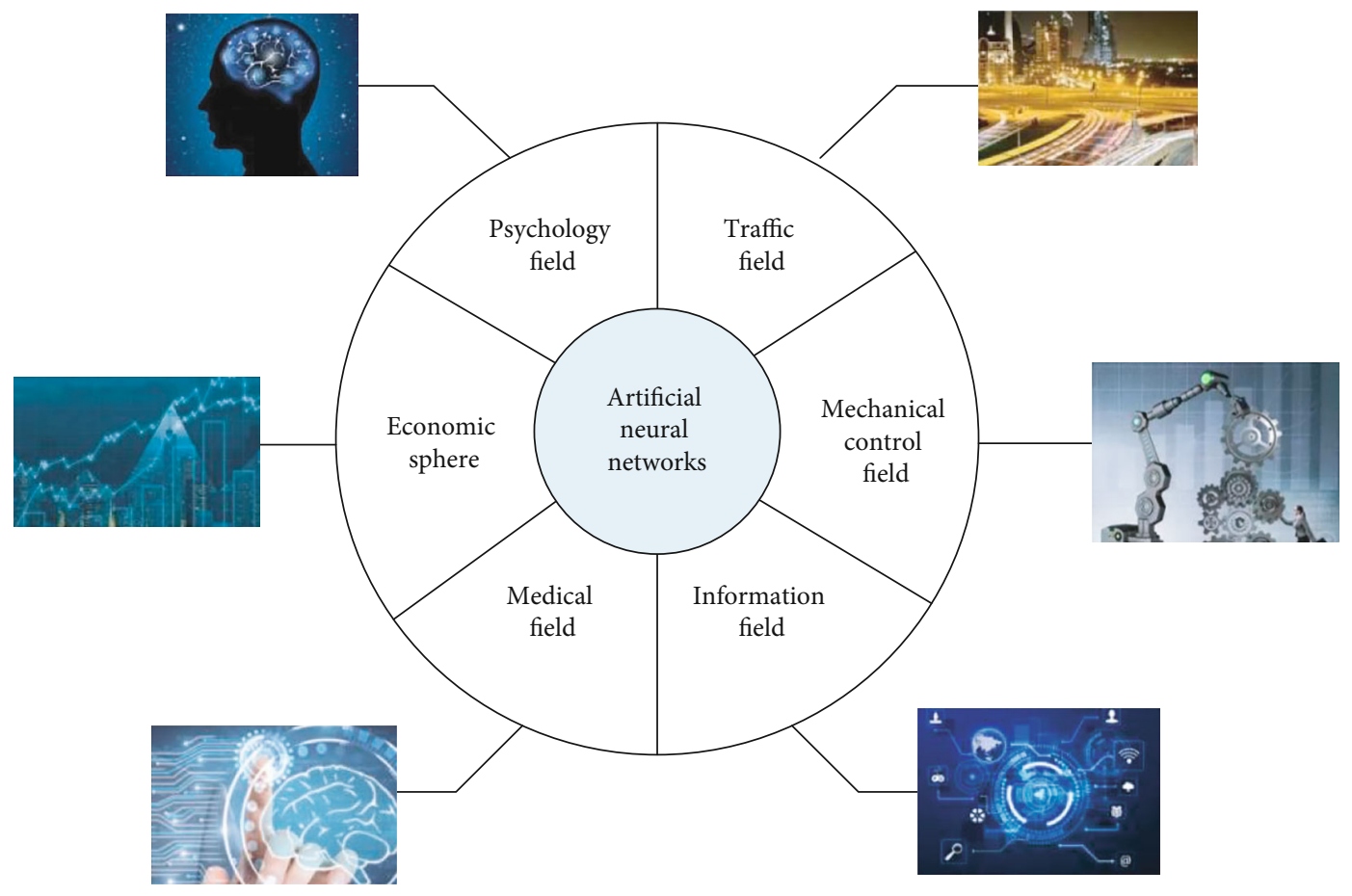

FIgURE 1: Application of ANN.

connection, a simulation of the combination of axons, synapses, and dendrites of brain neurons [19].

The deep neural network is a type of ANN. Combine lower-level functions to form a more abstract expression of higher-level functions. Data learning and detailed level feature modeling suitable for data features can be found.

The BP neural network is the direction propagation algorithm [20]. This algorithm can backpropagate calculation errors and conduct independent training based on multilayer feedforward networks. It is widely used in various fields. It is a simulation computing system that can realize autonomous learning and is especially good at data prediction.

The basic model of the BP neural network has a threelayer structure. The learning ability of the BP neural network is to get the nonlinear model by processing a large number of input variables and output variables, fitting the closest mapping relationship. Previous algorithms need to determine the weights of input variables, mapping relationships, mathematical expressions, etc., in advance, and the $\mathrm{BP}$ neural network model can complete this process autonomously, which is a "black box" model [21]. Because the transmission method of the BP neural network model is through reverse transmission, the weight and threshold of the model are continuously adjusted until the error is below the acceptable range.

ANN is a nonlinear, adaptive information processing system that simulates the neural network of the brain. This determines that the ANN includes the following characteristics and advantages of the BP neural network:

(1) Self-Adaptation, Self-Organization, and Self-Learning Ability. ANNs continuously modify and change the weights of connections between neurons through learning. Through this continuous learning process, the structure of the neural network is getting closer and closer to the actual situation. Self-adaptation is one of the important characteristics of neural networks, which refers to the ability of the system to adapt to environmental changes by changing its own performance. Self-organization is a process in which selfadaptability and self-organization ability are closely related to the neural network to realize self-adaptation. As the environment changes, the network can automatically adjust the structural parameters after specific perception and training and obtain the target output under the given input. In neural networks, training and learning are often mixed.

(2) Distributed Information Storage and Parallel Information Processing. ANN is a large-scale, highly parallel processing nonlinear system composed of multiple neuron connections. Therefore, the processing unit of neural networks has simple functions, but the number is large, and the network has rich functions. And it is faster. In order to parallelize the network structure, a distributed information storage structure is required, and certain parts of the network cannot store information. The parallelism and decentralization of the preservation and processing of neural network information will appear not only in time but also in space.

(3) High Degree of Nonlinearity, Nonprecision Calculation, and Good Fault Tolerance. Due to the parallel work of multiple neurons in the neural network, the neural network becomes very nonlinear. The fault tolerance of the network is mainly manifested in the decentralized storage structure. First, due to the distributed storage structure, even if some neurons are damaged, the overall performance of the system will not be affected, and the neuronal cells of the human 
brain will also be simulated. The same will not affect human brain function. In addition, the neural network can also use related functions to restore the memory of incomplete information, vague information, or deformed information, so as to correctly identify the information. Neural networks process continuous analog signals or incomplete and inaccurate fuzzy information. The result obtained is not a correct solution, but an approximate solution.

The BP algorithm has powerful nonlinear mapping function, strict export process, clear logic, elegant algorithm, and powerful general functions. However, the actual application program mainly has the following shortcomings.

(3)1. There Are Multiple Local Minimum Points. In the single-layer linear network, the average square error is a quadratic function with only one site, its hedge matrix is constant, the contour of the function is an ellipse, and the curvature change in a specific direction is constant. For the BP neural network of the multilayer network, the error function is not a quadratic function. Because the curvature varies greatly in the parameter space, it is difficult to determine the learning rate. For example, a larger learning rate is required in a flat area, and a higher curvature is required. Therefore, what may be caused is that the error function of the network may have multiple local minimum points, resulting in distortion of the shape. Some places may be relatively flat, and some places may be relatively curved. It is more difficult to deal with these areas by the steepest descent method. For example, there may be some samples whose gradient is close to 0 . Although these points are not close to the local minimum point, the network training will stop.

(3)2. Slow Convergence. Since the error function of the BP neural network activation function is not a quadratic function, the error surface has a flat area and a higher curvature area. Complicated error surfaces are difficult to deal with in relatively flat areas. Using the gradient descent method, there may be sample points with a gradient close to 0 . Although it is not a local minimum point at this time, it will also cause the training to stop. In this case, it takes time to exchange space for slowly exiting the area.

(3)3. The Network Structure Design Is Not Uniform. The structure of the BP neural network has been difficult to determine for a long time. One is that the number of hidden layers is difficult to determine; the other is that the number of hidden layer nodes is difficult to determine. Therefore, in practical applications, it can only be determined by experience and experiment. The problems to be solved are different, the mapping relationship is also different, and the network structure design has uncertainty.

2.2. Extraction of Surface Point Collection Information. The trimmed surfaces in the IGES file mainly include three types of revolving surfaces, column-labeled cylindrical surfaces, and NURBS surfaces. Since contact measurement requires point data, how to successfully extract the geometric infor-
TABle 1: Parameter data of the rotating surface.

\begin{tabular}{lccc}
\hline Index & Name & Type & Illustrate \\
\hline 1 & $L$ & Pointer & Pointer to $D E$ \\
2 & $Z$ & Pointer & Pointer to the bus entity $D E$ \\
3 & $F X$ & Real number & Starting angle in radians \\
4 & $T X$ & Real number & Final angle in radians \\
\hline
\end{tabular}

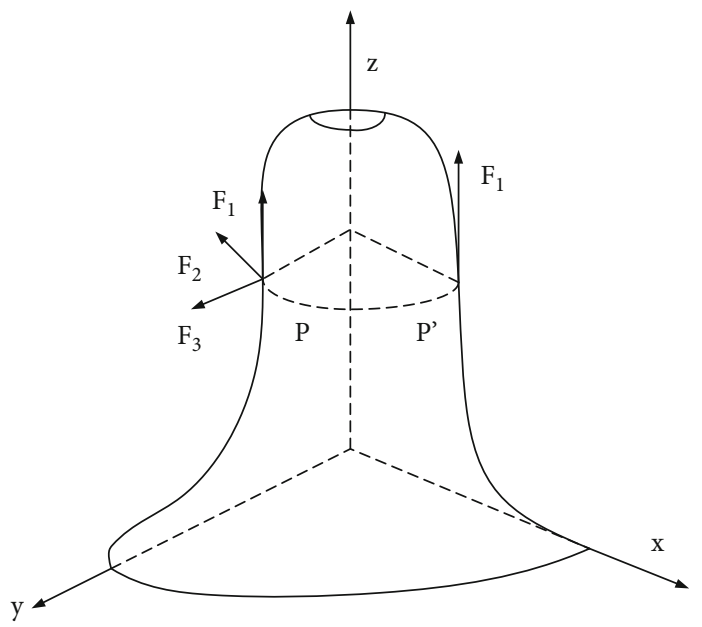

FIgURE 2: Schematic diagram of the normal direction of the rotating surface.

mation of the points on the three types of surfaces becomes an important prerequisite for measurement planning.

2.2.1. Rotating Surface. The surface of revolution is a curved surface swept by the generatrix rotating at a certain angle around the axis of rotation. The axis of rotation is a threedimensional line. The generatrix can be a straight line or a curve. The parameter data of the surface of revolution is shown in Table 1.

The default parameterization of the revolver is expressed as

$$
F(a, \mu)=T_{\mu}(Z(a))
$$

In the process of parameterization, the parameter of the bus bar is a parameter variable, and the rotation angle value $\mu$ is another parameter variable, among which $0 \leq a \leq 1, F X \leq \mu \leq R X$. If the bus bar is a straight line, the bus bar can be expressed as

$$
Z(a)=\left\{\begin{array}{l}
j=j_{0}+x * a, \\
i=i_{0}+y * a, \quad 0 \leq a \leq 1, \\
p=p_{0}+z * a .
\end{array}\right.
$$

If it is a NURBS curve, the bus can be expressed as

$$
Z(a)=\sum_{n=0}^{i} d_{n} I_{n, k}(a) .
$$


TABLe 2: Arbitrary parameter values of cylindrical surface.

\begin{tabular}{lccccccc}
\hline \multicolumn{2}{c}{ Parameter value } & \multicolumn{3}{c}{ Coordinate value } & \multicolumn{2}{c}{ Normal vector } \\
$N$ & $M$ & $X$ & $Y$ & $Z$ & $X$ \\
\hline 0.0345 & 3.7547 & -40.8919 & 3.1724 & 28.7724 & -16.3568 & 0 \\
0.1034 & 4.994 & 13.8975 & 17.5172 & 48.0298 & 5.559 & 0 \\
0.1379 & 3.5294 & -46.2867 & 24.6787 & 18.9088 & -18.5147 & 1.509 \\
0.2414 & 5.67 & 40.8919 & 46.2069 & 28.7724 & 16.3568 & 0 & 7.2119 \\
0.3106 & 3.4169 & -48.1191 & 60.5517 & 13.5851 & -19.2476 & 0 \\
\hline
\end{tabular}

TABle 3: Column-labeled cylinder reference data.

\begin{tabular}{lccc}
\hline Index & Name & Type & Illustrate \\
\hline 1 & $D E$ & Pointer & Pointer to the guideline entity $D E$ \\
2 & $L A$ & Real number & $X$ coordinate of bus end point \\
3 & $L B$ & Real number & $Y$ coordinate of bus end point \\
4 & $L C$ & Real number & $Z$ coordinate of bus end point \\
\hline
\end{tabular}

Among them, $d_{n}$ is the control vertex and $I_{n, k}(a)$ is the basis function.

Figure 2 shows the normal direction of the rotating surface, and Table 2 shows the parameter values of the cylindrical surface.

The normal direction of the rotating surface is as shown in the figure. Since there is no surface equation that can directly obtain the normal direction, the same principle as the coordinate of the point above is used to rotate the rotating axis until the starting point and the origin coincide. $Z$ axis coincides. At this time, the tangent vector of the point on the surface is obtained by using the derivative of the generatrix to obtain the normal vector, and then, the normal vector of the original point is obtained through the inverse matrix of the rotation matrix.

2.2.2. Column Standard Cylinder. The column standard cylindrical surface is a curved surface swept by the generatrix along the guideline. The generatrix is a line, and the guideline may be a straight line, arc, or NURBS curve. The start points of the generatrix and the origin of the guideline are the same point. The column-labeled cylinder parameter data is shown in Table 3.

The default parameters of the column label cylinder are expressed as

$$
F(n, m)=\left\{\begin{array}{l}
Z A(n)+m \cdot(L A-Z A(0)), \\
Z B(n)+m \cdot(L B-Z B(0)), \\
Z C(n)+m \cdot(L C-Z C(0))
\end{array}\right.
$$

Among them, $Z A(n), Z B(n), Z C(n)$ are the coordinate values of the guideline function when the parameter value is $n, n$ is the parameter of the guideline, $0 \leq n \leq 1, m$ are the parameters of the bus, and $0 \leq m \leq 1$.
Figure 3 shows a schematic diagram of the normal direction of the column-labeled cylinder, and Table 4 shows the arbitrary parameter values of the column-labeled cylinder.

2.2.3. NURBS Surface. The NURBS surface is a general analytical form of surface. Both planes and surfaces can be represented by NURBS surfaces. In IGES, except for revolving surfaces and list cylinders, other surfaces are represented by NURBS surfaces.

The rational fraction can be expressed as

$$
F(n, m)=\frac{\sum_{g=0}^{i} \sum_{h=0}^{j} I_{g, k}(n) I_{h, l}(m) w_{g, h} d_{g, h}}{\sum_{g=0}^{i} \sum_{h=0}^{j} I_{g, k}(n) I_{h, l}(m) w_{g, h}}, \quad 0 \leq n, m \leq 1 .
$$

They have node vectors towards $n$ and $m$, respectively,

$$
\begin{array}{r}
N\left[n_{0}, n_{1}, \cdots, n_{j+k+1}\right], \\
M\left[m_{0}, m_{1}, \cdots, m_{i+k+1}\right] .
\end{array}
$$

The $B$-spline basis function is defined like this:

$$
I_{g, k}(n)=\frac{n-n_{g-1}}{n_{g+k-1}-n_{g-1}} I_{g, k-1}(n)+\frac{n_{g+k}-n}{n_{g+k}-n_{g}} I_{g+1, k-1}(n)
$$

in

$$
I_{g, 0}(n)= \begin{cases}1, & n_{g-1} \leq n \leq n_{g} \\ 0, & \text { other }\end{cases}
$$

The discovery direction of the NURBS surface is shown in Figure 4. 


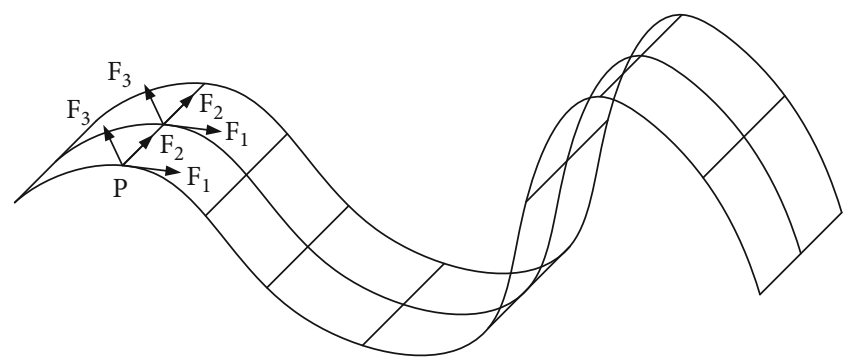

Figure 3: Schematic diagram of the normal direction of the column standard cylinder.

TABle 4: Arbitrary parameter values of column-labeled cylinder.

\begin{tabular}{lcccccccc}
\hline \multicolumn{2}{c}{$\begin{array}{c}\text { Parameter } \\
\text { value }\end{array}$} & \multicolumn{3}{c}{ Coordinate value } & \multicolumn{3}{c}{ Normal vector } \\
$N$ & $M$ & $X$ & $Y$ & $Z$ & $X$ & $Y$ & $Z$ \\
\hline 0.0345 & 0.6667 & -173.197 & 95.2837 & 151 & -1.5965 & 0.7612 & 0 \\
0.1379 & 0.1111 & -142.04 & 136.1794 & 21 & -0.5149 & 0.8073 & 0 \\
0.2414 & 0.3333 & -108.768 & 140.6955 & 73 & 0.2322 & 0.8675 & 0 \\
0.2759 & 0.1111 & -97.1109 & 136.4171 & 21 & 0.4068 & 0.8907 & 0 \\
0.2759 & 0.3333 & -97.1109 & 136.4171 & 73 & 0.4068 & 0.8907 & 0 \\
\hline
\end{tabular}

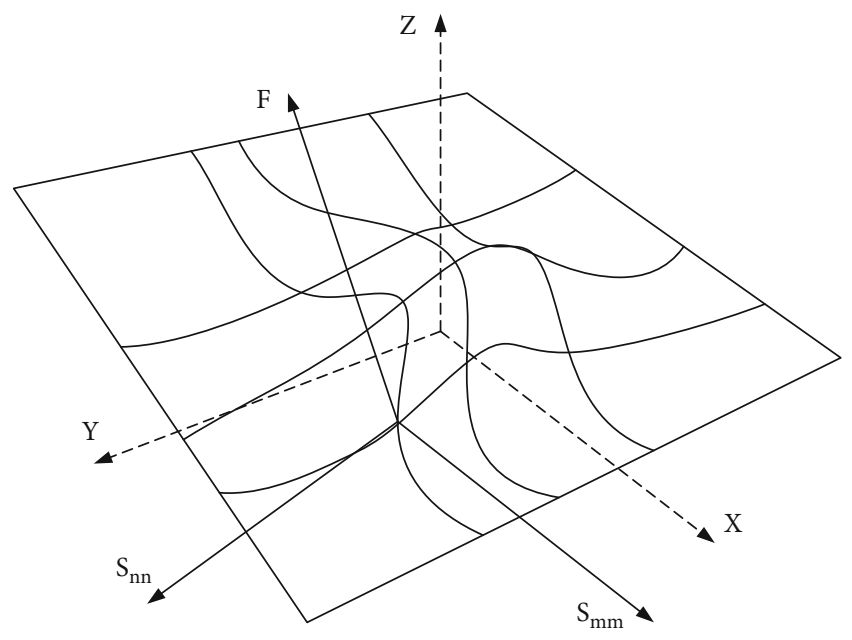

FigURE 4: Normal direction of NURBS surface.

First, use homogeneous coordinates to represent the NURBS surface, namely,

$$
F^{\omega}(n, m)=\sum_{g=0}^{i} \sum_{h=0}^{j} I_{g, p}(n) I_{h, p}(m) P_{g, h}^{\omega}
$$

in

$$
P_{g, h}^{\omega}=\left(\omega_{g, h} a_{g, h}, \omega_{g, h} b_{g, h}, \omega_{g, h} c_{g, h}, \omega_{g, h}\right) .
$$

$F^{\omega}(n, m)$ is the polynomial surface of the tensor integral section in the four-dimensional space.
Derivative formula using $B$-spline basis function:

$$
I_{g, p}^{\prime}=\frac{p}{n_{g+p}-n_{g}} I_{g, p}(n)+\frac{p}{n_{g+p+1}-n_{g+1}} I_{g+1, p-1}(n) .
$$

Calculate all the partial derivatives of the $B$-spline surface $F^{\omega}(n, m)$ up to order $d$ :

$\frac{\gamma^{k+l}}{\gamma^{k} n \gamma_{m}^{l}} F^{\omega}(n, m)=\sum_{g=0}^{i} \sum_{h=0}^{j} I_{g, p}^{(k)}(n) I_{h, p}^{(k)}(m) P_{n, m}, \quad k+l=0,1, \cdots, d$.

The calculation formula is

$$
\begin{gathered}
\frac{\gamma^{k+l}}{\gamma^{k} n \gamma_{m}^{l}} F^{\omega}(n, m)=\sum_{g=0}^{i-k} \sum_{h=0}^{j-l} I_{g, p-k}(n) I_{h, q-l}(m) P_{g, h}^{(k, l)} \\
P_{g, h}^{(k, l)}=(q-l+1) \frac{P_{g, h+1}^{(k, l-1)}-P_{g}^{(k, l-1)}}{m_{h+q+1}-m_{h+1}} .
\end{gathered}
$$

The partial derivative function of $F(n, m)$ can be calculated by the partial derivative of $F^{\omega}(n, m)$, making

$$
\begin{gathered}
F(n, m)=\frac{\omega(n, m) F(n, m)}{\omega(n, m)}=\frac{X(n, m)}{\omega(n, m)}, \\
X(n, m)=\sum_{g=0}^{i} \sum_{h=0}^{j} I_{g, k}(n) I_{h, l}(m) \omega_{g, h} P_{g, h} .
\end{gathered}
$$

Then, the partial derivative can be obtained as follows:

$$
\begin{gathered}
F_{n m}=\frac{X_{n m}-\omega_{n m} F-\omega_{n} F_{m}-\omega_{m} F_{n}}{\omega}, \\
F_{n n}=\frac{X_{n n}-2 \omega_{n} F_{n}-\omega_{n n} F}{\omega}, \\
F_{m m}=\frac{X_{m m}-2 \omega_{\mathrm{m}} F_{m}-\omega_{m m} F}{\omega} .
\end{gathered}
$$

Suppose the value of the parameter space corresponding to a point on the surface is $(n, m)$, then the normal vector of the curve from this point is

$$
\vec{S}=\vec{F}_{n n} \times \vec{S}_{m m} .
$$

Table 5 shows the arbitrary parameter values of the NURBS surface.

\subsection{Part Detection Method Based on Neural Network Model}

2.3.1. Basic Principles of Model Checking. Model checking is a method of verifying attributes based on one or more algorithms. For a given type of finite state system and a specific sequential logical expression that represents the nature or 
TABLE 5: Arbitrary parameter values of NURBS surface.

\begin{tabular}{lcccccc}
\hline \multicolumn{2}{c}{ Parameter value } & \multicolumn{3}{c}{ Coordinate value } & \multicolumn{2}{c}{ Normal vector } \\
$N$ & $M$ & $X$ & $Y$ & $X$ & $Y$ \\
\hline 0.1379 & 0.3333 & -69.7861 & 97.6182 & 207.3362 & 0 & -1.0623 \\
0.2759 & 0.2222 & -105.589 & 207.6449 & 221.7298 & 0 & 0.2156 \\
0.2759 & 0.4444 & -33.983 & 207.6449 & 221.7298 & 0 & 0.2156 \\
0.3103 & 0.8889 & 109.2291 & 234.7555 & 218.5632 & 0 & 0.4352 \\
0.4828 & 0.4444 & -33.983 & 366.733 & 180.6447 & 0 & 2.8287 \\
\hline
\end{tabular}

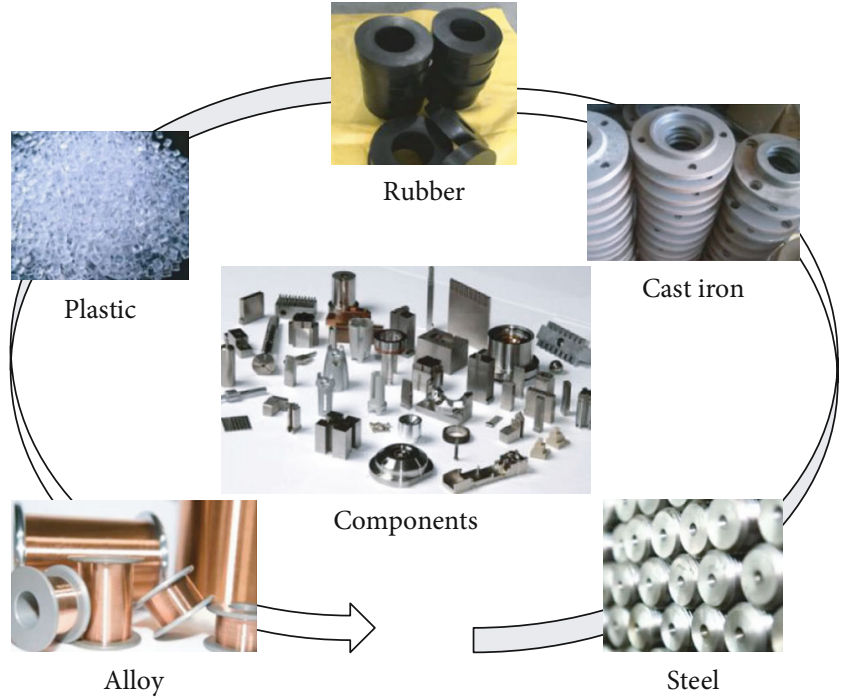

Figure 5: Types of part materials.

specification of the system, model checking can determine whether the given system meets the logical description of the formula nature or specification, thereby verifying the finite state. Shown in Figure 5 are the types of part materials.

Compared with other methods, the model checking method has two significant advantages:

(1) It is fully automated, and this method does not require users to have professional mathematical knowledge and experience. Anyone who can simulate and verify a design is fully capable of using model checking to verify the same design. Compared with the current actual verification methods, model checking can be regarded as a more accurate simulation tool

(2) If it is detected that the design does not meet the expected characteristics, the model check can give a counterexample, which can show how the system's actions are violated. This feedback method on defects helps to understand the real cause of the error, provides clues to correct the problem, and can help the verifier locate the key to the problem and then improve the system

The verification of system design using model checking includes three main steps: system modeling, characterization, and input to the model checker for verification:
(1) Modeling. The first step is to transform the design into a form acceptable to the model checker. In most cases, this is just a compilation process, but due to the limitations of verification time and computer memory, abstract techniques may need to be used to reduce the formal model, remove irrelevant factors, and abstract the model as much as possible

(2) Property Specification. Before verification, it is necessary to tell the model checker the properties that the designed model should satisfy. For the verification of hardware and software systems, temporal logic is usually used to describe the nature of the system. This logical expression can indicate changes in the state of the system's behavior according to changes in time. Although model checking provides a method to verify whether the model to be tested satisfies the given properties, this method cannot completely guarantee that the characterization can be accurately expressed, nor can it be guaranteed to describe all the properties that the system to be verified should meet

(3) Verification. The verification process of model checking should be completely automatic under ideal conditions. However, in practice, the verification result cannot be automatically analyzed, and it requires human intervention. When a failed verification result is obtained, a counterexample can usually be given so that the designer can determine the specific location of the violation. After analyzing the counterexample to correct the system design, the model needs to be checked again and reverified until the verification is passed

2.3.2. Principles of Quality Control. The control chart is a kind of control chart that is often used for control in mathematical statistics. It is composed of a center line and upper and lower control lines and is drawn according to the sequence of product data entry. The data of the control chart can be either count data or meter data. As shown in Figure 6, CL represents the central control line, UCL represents the upper control line, and LCL represents the lower control line. As long as the measurement data of the process product falls between the upper and lower lines, it means that there is no problem with the quality. The upper and lower lines are the maximum and minimum allowable errors specified by the standard. If the allowable error is zero, then the three lines will be integrated. The product data that is not online 


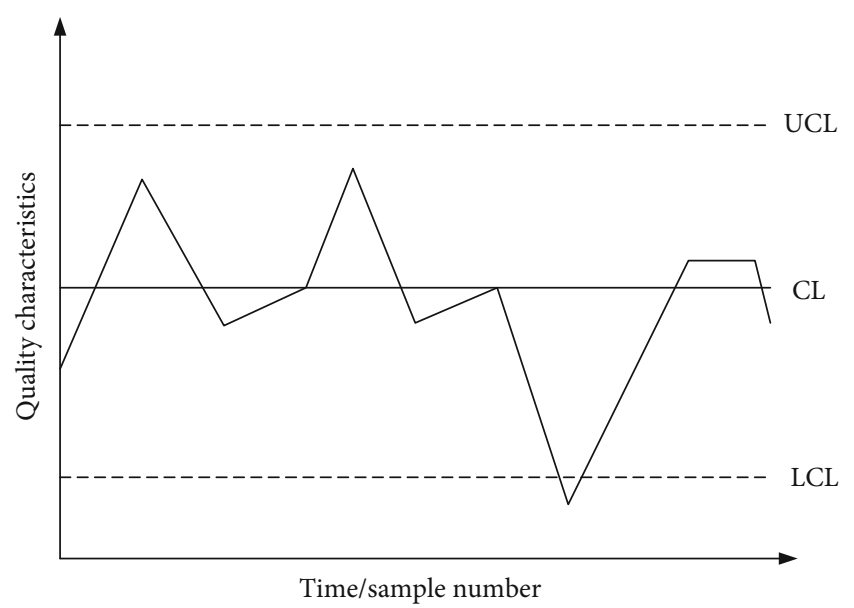

FIgURE 6: Schematic diagram of quality control line.

indicates that the product has quality problems and needs to be overhauled and cannot enter the next process before passing the test. Product feature data that exceeds the upper and lower control lines indicate that their quality is unqualified. Collect the data of these products through the database and then find the products of each process through the barcode. This completes the preliminary work of control and makes subsequent corrective measures easier.

\section{Neural Network Model Part Manufacturing Quality Inspection Experiment}

3.1. Experiment of Welding Basic Formation Parameters. In the process of arc additive manufacturing, the morphology of the single-pass weld is the basis of metal accumulation and forming. The study of the forming law of the singlepass weld plays an important role in the dimensional accuracy control of the forming of complex parts. The welding experiment of a single-pass weld on the substrate mainly depends on the welding current, shielding gas, welding speed, dry wire elongation, wire feeding speed, welding voltage, and other parameters, in order to be able to accurately control the single-pass weld. It is necessary to study the influence of the main process parameters in the welding process on the melting width and the reinforcement and to summarize the change rules. The three welding parameters of welding current, welding speed, and wire feeding speed have a relatively large variation range, which is easy to adjust and controllable in the experiment. These three parameters can be used as the main variables to study their effects on the shape of the weld.

The Fronius digital welding machine in the experimental system has a built-in unified mode; that is, only the welding current needs to be controlled, and the wire feeding speed and welding voltage can be controlled indirectly by adjusting the welding current. Therefore, in this experiment, the welding current and welding speed are used as variables, and the weld width and reinforcement are used to express the welding seam forming accuracy, so as to study the influence of different welding process parameters on the welding seam geometric parameters.
For a single-pass weld, when the current is small and the welding speed is fast, the heat input is too small, which makes it difficult for the metal welding wire to melt, and the weld cannot be formed continuously. When the current is large and the welding speed is slow, too much heat input will cause the welding seam to be overburned and a good shape cannot be achieved. Considering the efficiency and quality of weld cladding, the welding current and welding speed should be selected within an appropriate range. According to the preliminary exploratory test, the range of welding current is $100 \mathrm{~A}-350 \mathrm{~A}$, and the range of welding speed is $2-10 \mathrm{~mm} / \mathrm{s}$.

A single-pass deposition experiment of MIG welding was carried out on the substrate. The welding current increased from $100 \mathrm{~A}$ to $350 \mathrm{~A}$, and the welding speed remained unchanged at $6 \mathrm{~mm} / \mathrm{s}$. Then, adjust the welding speed to gradually increase from $3 \mathrm{~mm} / \mathrm{s}$ to $9 \mathrm{~mm} / \mathrm{s}$, and the welding current remains unchanged at $225 \mathrm{~A}$. A singlepass deposition experiment of MIG welding is performed on the substrate. The experimental results are shown in Figure 7.

According to the analysis result of Figure 7, when the welding current increases, the melting width and the surplus height of the single-pass weld will increase correspondingly. This is because the wire feeding speed will increase with the increase of the current, and the amount of metal deposited in a unit time will also increase, resulting in an increase in the volume of a single weld. At the same time, the increase of the welding current will increase the heat input, and the molten metal will be easier to spread on the substrate, so the increase of the current has more influence on the melting width than the remaining height. When the value of the welding current is less than $175 \mathrm{~A}$, the increase in the weld width of the weld is relatively obvious. At this time, the heat input increases quickly and the weld size changes quickly. When the welding current is increased from $175 \mathrm{~A}$ to $300 \mathrm{~A}$, the range of change in melt width decreases. When the current is greater than $300 \mathrm{~A}$, the impact on the increase in weld width is maximized. The influence of welding current on the change of weld reinforcement is relatively small. When the welding current continues to increase, the change of the reinforcement is not large. There is a strict negative correlation between welding speed and weld width and reinforcement. This is because when the welding speed increases, the heat input of the welding arc per unit time will be reduced accordingly, the temperature of the molten pool will also be correspondingly reduced, the wetting time of the metal on the substrate will be reduced, and the fluidity of the molten metal will deteriorate, so the weld width will decrease. In addition, the acceleration of welding speed will also reduce the amount of deposited metal per unit area, so the value of melting width and excess height in the size of a single weld will be reduced accordingly.

3.2. Robustness Analysis. In order to verify the image robustness analysis capability of the part quality inspection method designed in this article combined with the ANN model, this article designed experiments on 4 sets of models, and the results are shown in Figure 8. 

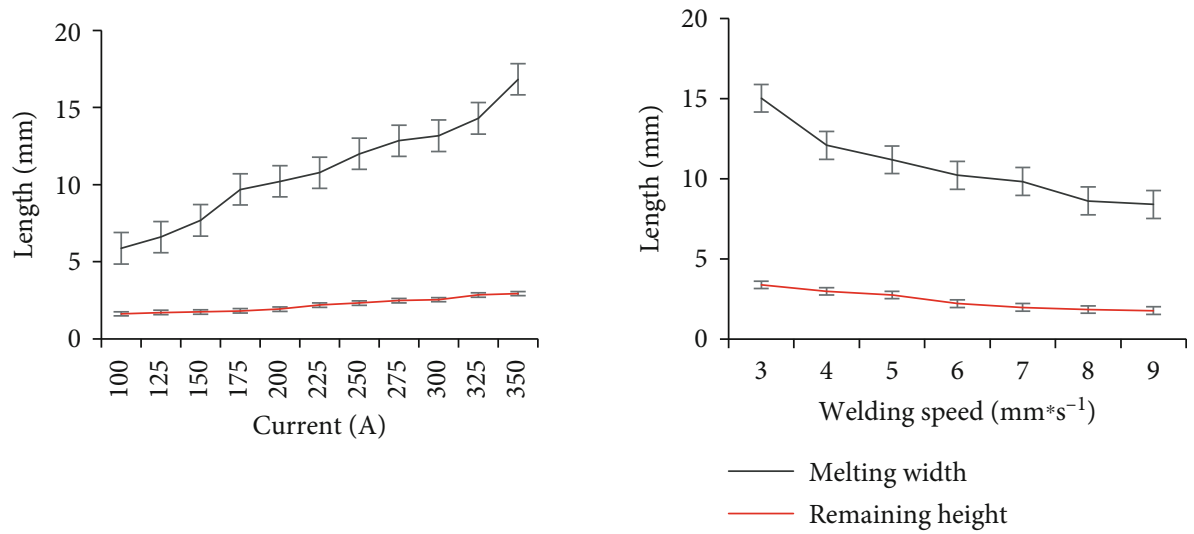

(a) The influence of different currents on the weld

(b) The influence of different welding speeds on the weld

Figure 7: The influence of different currents and welding speeds on the weld.

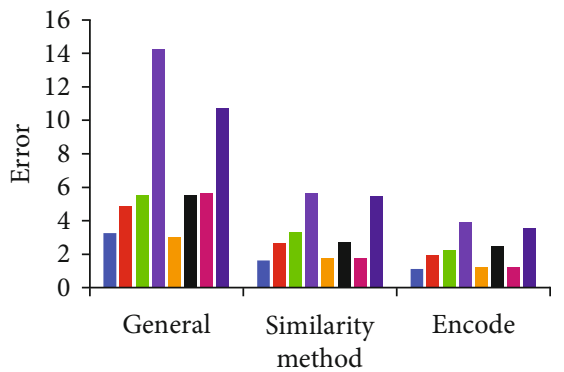

(a) Comparison of the average pixel error of the image under different lights

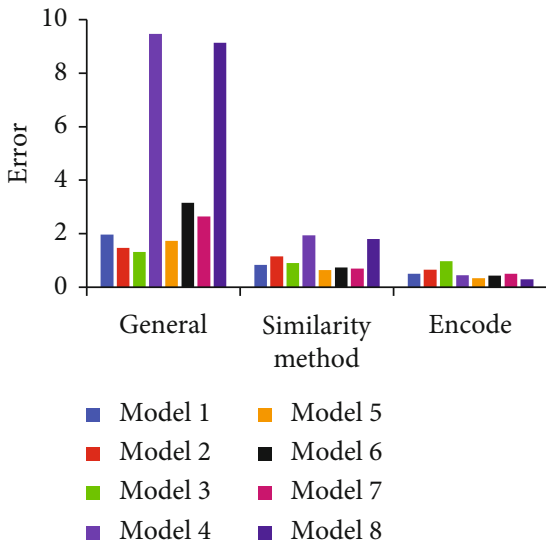

(b) Comparison of the average pixel error of the circle radius under different lights

FIGURE 8: Comparison of the average pixel error of the image and circle radius under different light conditions of the robustness enhancement algorithm.

It can be analyzed from the figure that if the original image is directly used for matching under two different lights, the average matching error is very large; using the similarity algorithm and image coding algorithm to preprocess the original image can greatly reduce the matching error. In addition, the average matching error of image coding is smaller than that of the similarity algorithm. After similarity calculation and detection, the average error of the circle radius is about 2 pixels, and the average error of the circle radius obtained by the image coding detection is within 1 pixel, which has a good detection effect.

\section{The Inspection Results of the Manufacturing Quality of the Neural Network Model Parts}

4.1. The Quality Inspection Test Analysis of the Outer Diameter of the Parts. The rod end diameter, the proximal rod end diameter, the rod diameter, and the disc diameter of the parts are regarded as a type of measurement. The dimensional accuracy of these diameters is relatively high, and the method of linear fitting or the smallest circum- scribed rectangle is generally used. The dimensional accuracy obtained by the method measurement is generally not too high, so the realization process of this type of measurement is as follows: the first step is to select the range to be detected; first, use the Canny operator for rough edge positioning, and then, use the improved binomial interpolation to perform edge precise positioning to obtain subpixel edge coordinates; the second step selects the subpixel coordinate points of the leftmost and rightmost edges; the third step selects multiple pairs of left and right edge coordinates corresponding to the $x$ value subtraction, and take the average value as the number of pixels of the diameter is multiplied by the calibration coefficient to get the actual measured diameter; finally, the actual measured value is compared with the standard value to determine whether the diameter is within the allowable range of error, as shown in Figure 9 for the detection result.

It can be seen from the figure that the error requirement is $\pm 0.1 \mathrm{~mm}$, and the test results are all compounded by this standard, so the test method can be applied in practice. 


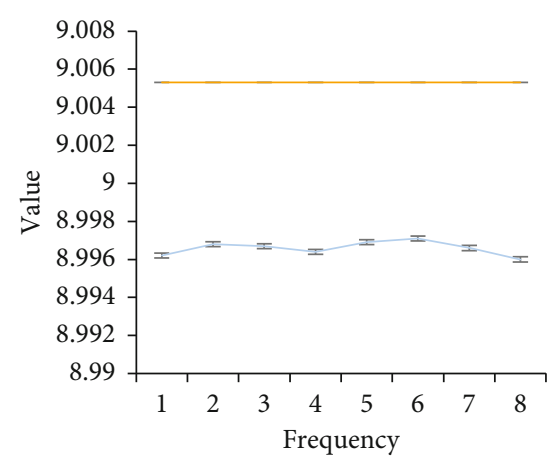

(a) The measurement result of the diameter of the part rod

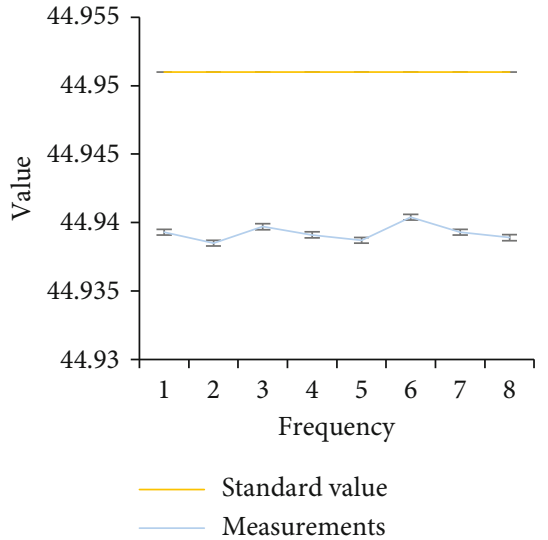

(b) The measurement result of the diameter of the part disc

FIGURE 9: Inspection results of the outer diameter of parts.

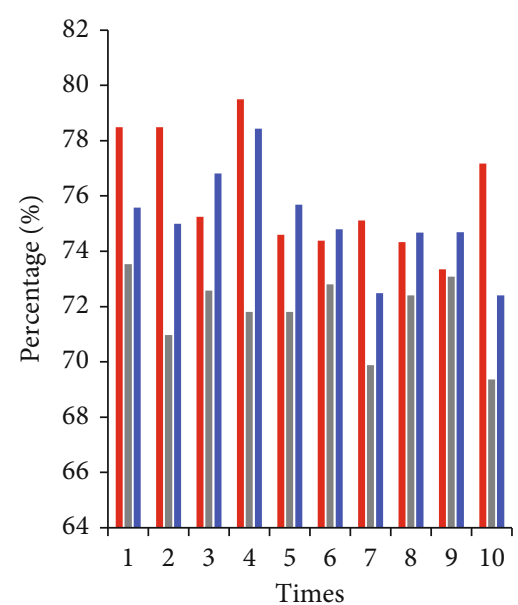

(a) Traditional part quality inspection results

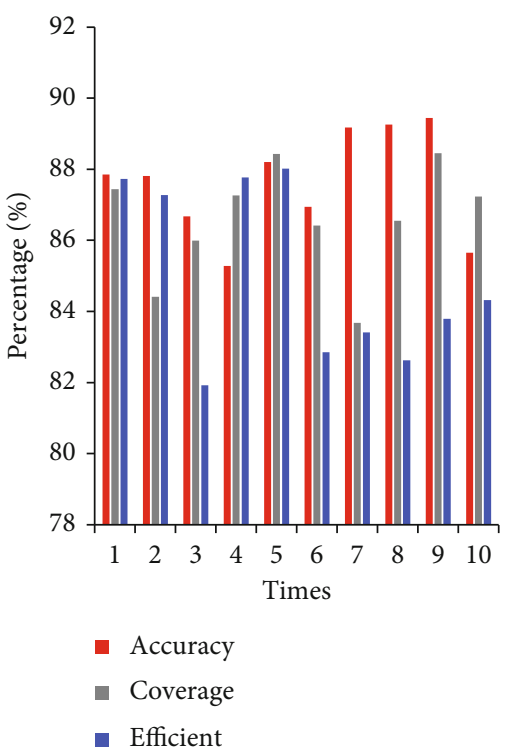

(b) Model-based part quality inspection results

Figure 10: Comparison results with traditional part quality inspection path.

4.2. Comparative Experimental Analysis with Traditional Detection Path. After combining the ANN model and the part quality inspection method of the test experiment, this paper designs an optimized part quality inspection path. In order to verify the accuracy of the path, this paper compares the optimized part quality inspection path with the traditional part quality inspection path; the experimental results are shown in Figure 10.

It can be seen from the figure that the accuracy of the optimized path for part manufacturing quality inspection based on the ANN model is as high as $85 \%-90 \%$, while the accuracy of the traditional part manufacturing quality inspection path is only $73 \%-80 \%$. Therefore, the new part quality inspection path proposed in this paper can improve the accuracy rate of $5 \%-17 \%$, the coverage rate of $9 \%-20 \%$, and the efficiency of $3 \%-17 \%$. This is a very critical optimization path for the part manufacturing industry. Based on this new part quality inspection path, the quality of part manufacturing and the efficiency of part manufacturing can be improved, and it is a brand-new reform for the quality inspection of part factories.

\section{Conclusions}

This article mainly studies the optimization of the part manufacturing quality inspection path based on the model, so this article uses the BP neural network in the neural network model to perform deep learning optimization on the part quality inspection system, then accelerates the efficiency of part recognition through the extraction of surface point collection information, and finally combines the basic principles of model checking and quality control principles to optimize the path. Then, through the welding basic formation parameter experiment and robustness analysis to verify 
the influencing factors of the welding process and the specific results of the image processing, combine the data generated by the experiment to analyze the quality inspection test of the outer diameter of the part, and finally, pass the result of the traditional part quality inspection. By comparison, the new part quality inspection path can improve the accuracy rate of $5 \%-17 \%$, the coverage rate of $9 \%-20 \%$, and the efficiency of $3 \%-17 \%$ compared with the traditional part quality inspection path.

\section{Data Availability}

No data were used to support this study.

\section{Conflicts of Interest}

There are no potential competing interests in our paper. And the authors have seen the manuscript and approved to submit it to your journal. I confirm that the content of the manuscript has not been published or submitted for publication elsewhere.

\section{References}

[1] H. Chen, Y. Lu, and L. Tu, "Fault identification of gearbox degradation with optimized wavelet neural network," Shock and Vibration, vol. 20, no. 2, p. 262, 2013.

[2] C. $\mathrm{Li}$ and P. Xu, "Application on traffic flow prediction of machine learning in intelligent transportation," Neural Computing and Applications, vol. 33, no. 2, pp. 613-624, 2021.

[3] M. Zheng and K. Wu, "Smart spare parts management systems in semiconductor manufacturing," Industrial Management \& Data Systems, vol. 117, no. 4, pp. 754-763, 2017.

[4] B. R. Maria, "Printing spare parts through additive manufacturing: legal and digital business challenges," Journal of Manufacturing Technology Management, vol. 29, no. 6, pp. 958-982, 2018.

[5] E. Y. Chen, "A neural network model of cortical information processing in schizophrenia II - role of hippocampal-cortical interaction: a review and a model," The Canadian Journal of Psychiatry, vol. 40, no. 1, pp. 21-26, 1995.

[6] Y. Cui, S. Ahmad, and J. Hawkins, "Continuous online sequence learning with an unsupervised neural network model," Neural Computation, vol. 28, no. 11, pp. 2474-2504, 2016.

[7] B. H. Truong, P. Nallagownden, K. H. Truong, R. Kannan, D. N. Vo, and N. Ho, "Multi-objective search group algorithm for thermo-economic optimization of flat-plate solar collector," Neural Computing and Applications, vol. 33, no. 19, pp. 12661-12687, 2021.

[8] Y. Wang, D. Yang, X. Zhang, and Z. Chen, "Probability based remaining capacity estimation using data-driven and neural network model," Journal of Power Sources, vol. 315, no. may 31, pp. 199-208, 2016.

[9] R. Peletz, J. Kisiangani, M. Bonham et al., "Why do water quality monitoring programs succeed or fail? A qualitative comparative analysis of regulated testing systems in sub-Saharan Africa," International Journal of Hygiene and Environmental Health, vol. 221, no. 6, pp. 907-920, 2018.
[10] K. Naus, "Drafting route plan templates for ships on the basis of AIS historical data," Journal of Navigation, vol. 73, no. 3, pp. 1-20, 2019.

[11] M. Nahrendorf and F. K. Swirski, “Abandoning M1/M2 for a network model of macrophage function," Circulation Research, vol. 119, no. 3, pp. 414-417, 2016.

[12] S. M. Blair, C. D. Booth, G. Williamson, A. Poralis, and V. Turnham, "Automatically detecting and correcting errors in power quality monitoring data," IEEE Transactions on Power Delivery, vol. 32, no. 2, pp. 1005-1013, 2017.

[13] V. L. Corbit, T. C. Whalen, K. T. Zitelli, S. Y. Crilly, J. E. Rubin, and A. H. Gittis, "Pallidostriatal projections promote $\beta$ oscillations in a dopamine-depleted biophysical network model," Journal of Neuroscience, vol. 36, no. 20, pp. 5556-5571, 2016.

[14] E. Busink and S. Postma, "Provincial soil-quality monitoring networks in the Netherlands as an instrument for environmental protection," Geologie en Mijnbouw, vol. 79, no. 4, pp. 429-440, 2000.

[15] G. Di Leo, C. Liguori, A. Pietrosanto, and P. Sommella, "A vision system for the online quality monitoring of industrial manufacturing," Optics \& Lasers in Engineering, vol. 89, no. feb., pp. 162-168, 2017.

[16] L. Ding, K. Li, Y. Zhou, and P. E. D. Love, "An IFC-inspection process model for infrastructure projects: enabling real- time quality monitoring and control," Automation in Construction, vol. 84, no. dec., pp. 96-110, 2017.

[17] X. Xu, D. Cao, Y. Zhou, and J. Gao, "Application of neural network algorithm in fault diagnosis of mechanical intelligence," Mechanical Systems and Signal Processing, vol. 141, article 106625, 2020.

[18] J. He, L. Xu, P. Wang, and Q. Wang, "A high precise E-nose for daily indoor air quality monitoring in living environment," Integration, vol. 58, no. jun., pp. 286-294, 2017.

[19] M. Hecquet and P. Brochet, "Time variation of forces in a synchronous machine using electric coupled network model," IEEE Transactions on Magnetics, vol. 34, no. 5, pp. 3214-3217, 1998.

[20] H. Li, C. S. Boling, and A. J. Mason, "CMOS Amperometric $\mathrm{ADC}$ reconfigurable resonant regulating rectifier with primary equalization for extended coupling- and loading-range in bioimplant wireless power transfer," IEEE Transactions on Biomedical Circuits and Systems, vol. 10, no. 4, pp. 1-11, 2016.

[21] B. M. Lesht, R. P. Barbiero, and G. J. Warren, "Using satellite observations to assess the spatial representativeness of the GLNPO water quality monitoring program," Journal of Great Lakes Research, vol. 44, no. 4, pp. 547-562, 2018. 